\title{
Distributed optical fiber temperature sensor using only anti-Stokes Raman scattering light in a loop configuration
}

\author{
Marcelo A. Soto, Alessandro Signorini, Tiziano Nannipieri, Stefano Faralli, Gabriele Bolognini*, \\ and Fabrizio Di Pasquale \\ Scuola Superiore Sant'Anna, via G. Moruzzi 1, 56124 Pisa, Italy. \\ *E-mail: g.bolognini@sssup.it
}

\begin{abstract}
In this paper we demonstrate distributed Raman temperature sensing (RDTS) in a loop scheme employing anti-Stokes light intensity only. Using a single-channel receiver and anti-Stokes traces measured in loop configuration, we implement RDTS with inherent compensation of fiber wavelength-dependent losses, as well as local external perturbations. Experimental results show a signal-to-noise ratio enhancement with respect to a standard RDTS in loop configuration, providing a robust and reliable high-performance sensor for long sensing ranges.
\end{abstract}

Keywords: Raman scattering, fiber optic sensors, temperature sensing.

\section{INTRODUCTION}

Raman-based distributed temperature fiber sensors (RDTS) have been studied for many years [1,2] and, owing to the well-known advantages over their electrical counterparts, they have found successful applications in many different areas, such as fire detection, power cable monitoring and leakage detection. Most of developed Raman temperature sensors are based on simultaneous measurements of Raman anti-Stokes (AS) and Stokes (S) (or Rayleigh) backscattered components in a single-ended fiber configuration [2,3]. Although they represent the most common solution in long range applications due to their extended sensing distance, sensors based on single-ended fiber configuration are intrinsically affected by variations of the wavelength-dependent losses (WDL) (also known as differential losses) of the fiber. In practical RDTS systems, both WDL and local losses must be cancelled out; this can be effectively achieved by employing a loop configuration technique, based on a double-ended scheme, in which AS and S traces are acquired in both forward and backward directions and then properly averaged [4]. For single-ended schemes, the use of AS light only has been proposed in [5], together with a suitable reflective mirror at the far fiber-end. However, the four-fold optical path undergone by the light pulse and its scattering makes this technique not suitable for long-range sensing due to very high experienced losses. Moreover, the use of a strongly reflective mirror would make the system sensitive to multiple optical reflections (ghosts) with unknown connector conditions.

In this paper we perform long-range Raman temperature sensing based on a loop-scheme, with inherent WDL correction, using AS-backscattered light only, and employing a simple, single-channel receiver. Experimental results also point out an enhanced temperature resolution, compared to standard RDTS, due to the noise reduction in temperature derivation based on AS light only. Such a technique can allow for simpler and cost-effective long-range RDTS systems characterized by WDL-independent and ghost-insensitive temperature measurements.

\section{THEORY}

RDTS systems are based on the temperature dependence of the spontaneous Raman anti-Stokes backscattered light, where the spatial information is obtained from a short pulse of light that is launched into the optical fiber. While the optical pulse propagates along the fiber, it generates two Raman backscattered components (Stokes and anti-Stokes lights), which are measured as a function of time at the receiver by using optical time-domain reflectometry (OTDR) techniques [1,3]. In order to distinguish temperature variations in the AS trace from fiber attenuation and local losses (such as splices, connectors and bending losses), the AS intensity must be normalized by a temperature-independent signal, such as the Raman Stokes [1] or Rayleigh intensity [3]. Since this normalization has to be done at every fiber position, a correction of the differential group velocity is also required. Thus, for instance, the relationship between the fiber temperature $(T)$ and the ratio $R(z, T)$ of anti-Stokes $\left(I_{A S}\right)$ over Stokes $\left(I_{S}\right)$ intensities can be expressed as:

21st International Conference on Optical Fiber Sensors, edited by Wojtek J. Bock, Jacques Albert, Xiaoyi Bao, Proc. of SPIE Vol. 7753, 77539U · ( 2011 SPIE · CCC code: 0277-786X/11/\$18 · doi: 10.1117/12.886031 


$$
R(z, T)=\left(\frac{\lambda_{S}}{\lambda_{A S}}\right)^{4} \exp \left(\frac{-h \Delta v}{k T(z)}\right) \exp \left\{-\int_{0}^{z}\left[\alpha_{A S}(\xi)-\alpha_{S}(\xi)\right] d \xi\right\},
$$

where $\lambda_{S}$ and $\lambda_{A S}$ are the Stokes and anti-Stokes wavelengths; $\alpha_{S}$ and $\alpha_{A S}$ are the fiber attenuation coefficients at $\lambda_{S}$ and $\lambda_{A S}$ respectively; $\Delta v$ is the frequency separation between AS and pump signal, $h$ is the Planck constant and $k$ is the Boltzmann constant [4]. If the differential WDL $\left(\alpha_{A S}-\alpha_{S}\right)$ is characterized as a function of the distance, the corresponding exponential factor of Eq. (1) can be corrected. However, in most harsh environments the optical fiber is exposed to conditions that can spectrally change the fiber attenuation properties. In such cases, the WDL (differential loss) is not constant in time, making single-ended RDTS inappropriate for such applications. In fact, it has been shown that the fiber loss can be significantly modified by external hydrogen-rich, hot or humid environments inducing wavelength-dependent fiber properties degradation. This is also the case e.g. of nuclear applications, where the presence of ionizing radiation increases the optical fiber attenuation with time. Hence, the ratio anti-Stokes/Stokes or anti-Stokes/Rayleigh is expected to change (more or less rapidly) during the sensor lifetime due to the ageing, leading to significant errors in temperature estimation when no further calibration is performed [4]. This actually appears as a gradual deviation of the detected temperature traces with respect to the real values, an effect that increases with the sensing distance turning out to be critical especially at long sensing ranges.

In order to compensate differential WDL several methods have been recently proposed, most commonly employing: $i$ ) the AS signal reflected from a mirror at the far fiber-end (providing a suitable solution for short-to-medium sensing ranges only, due to 4-fold loss increase in the optical path) [5], ii) using two light sources with different wavelengths [6], and, most notably, iii) using a sensing fiber in loop configuration (requiring the access to both fiber ends). The latter technique provides a robust and reliable method to compensate differential WDL, through the calculation of geometric means associated to AS and S lights measured in both forward and backward directions. In such a way, the effects of fiber attenuation and local losses are inherently cancelled out in temperature estimation through the $\mathrm{AS} / \mathrm{S}$ ratio, leading to auto-calibrated measurements that do not depend on optical fiber loss variations during the sensor lifetime.

The technique proposed in this paper allows for RDTS loop schemes based on AS light detection only. The anti-Stokes intensity traces are measured in both forward $\left(I^{F o r}\right)$ and backward $\left(I^{B a c k}\right)$ directions, and their relation to the fiber temperature can be expressed as:

$$
\begin{aligned}
& I_{A S}^{F o r}(z)=C_{A S_{-} F o r} \exp \left(-\int_{0}^{z}\left[\alpha_{A S}(\xi)+\alpha_{P}(\xi)\right] d \xi\right)\left[\exp \left(\frac{h \Delta v}{k T(z)}\right)-1\right]^{-1}, \\
& I_{A S}^{B a c k}(z)=C_{A S_{-} B a c k} \exp \left(-\int_{z}^{L}\left[\alpha_{A S}(\xi)+\alpha_{P}(\xi)\right] d \xi\right)\left[\exp \left(\frac{h \Delta v}{k T(z)}\right)-1\right]^{-1},
\end{aligned}
$$

where $\alpha_{P}$ is the fiber attenuation at the pump wavelength, and $C_{A S_{-} \text {For }}$ (forward direction) and $C_{A S_{-} \text {Back }}$ (backward direction) are constant parameters accounting for the input pump power, the receiver response and the Raman crosssection at $\lambda_{A S}$ wavelength. Note that a suitable normalization is necessary when dealing with time-dependent laser power fluctuations, since this issue cannot be neglected when using the AS light only in the temperature estimation (differently from the $\mathrm{AS} / \mathrm{S}$ ratio case, which is inherently insensitive to laser fluctuations). In the AS-only approach several effective normalization schemes can be implemented to simply address this issue, namely $i$ ) the use of a temperature-controlled reference fiber [4], ii) by measuring the launched optical pulse power through an optical tap coupler or, iii) by using a laser current monitor. In our experiments, we used a built-in current monitor in the pulsed laser source for AS trace normalization, providing a simple and cost-effective solution for trace calibration.

The AS intensity in loop configuration $I_{A S \text { Loop }}(z)$ can then be obtained from the geometric mean of the normalized singleended AS traces $I_{A S_{-} n}(z)$ in both forward and backward directions according to

$$
I_{A S_{-} L o o p}(z)=\sqrt{I_{A S_{-} n}^{F o r}(z) \cdot I_{A S_{-} n}^{\text {Back }}(z)}=C_{A S_{-} \text {Loop }}\left[\exp \left(\frac{h \Delta v}{k T(z)}\right)-1\right]^{-1} \exp \left(-\frac{1}{2} \int_{0}^{L}\left[\alpha_{A S}(\xi)+\alpha_{P}(\xi)\right] d \xi\right)
$$

where $C_{A S \text { Loop }} \propto\left[C_{A S \text { For }} \cdot C_{A S \text { Back }}\right]^{1 / 2}$ is a constant factor that depends on the Raman cross-section of the fiber and is normalize $\bar{d}$, as mentioned before, to take into account fluctuations of laser peak power. Note that the exponential factor in Eq. (4), expressing the effects of WDL, is a constant parameter that does not depend on the fiber position, contrarily to the $z$-dependent exponential term in Eq. (1). Moreover, to take into account the effects of Raman scattering crosssections, a calibration is performed for different fiber spools employing a known reference temperature $\left(T_{\text {ref }}\right)$, similarly to 
standard RDTS systems [4-6]. Finally, the WDL-corrected temperature profile $T(z)$ can be derived from previous equations leading to the following expression:

$$
T(z)=\left\{\frac{k}{h \Delta v} \ln \left[\frac{I_{A S_{-} \text {Loop }}\left(z, T_{\text {ref }}\right)}{I_{A S_{-} \text {Loop }}(z, T)}\left[\exp \left(\frac{h \Delta v}{k T_{\text {ref }}}\right)-1\right]+1\right]\right\}^{-1} .
$$

Based on Eq. (5), a self-calibrated temperature sensor for long sensing distances can then be implemented using AS light only, without employing the Stokes light (and hence, avoiding the use of both double receiver and Stokes optical filter). Additional advantages of the proposed scheme are related to the fact that group velocity correction, required in the AS/S ratio approach, can now be avoided, and to the better achievable temperature resolution, with respect to standard loopRDTS systems with same measurement time, due to use of AS light only.

\section{EXPERIMENTAL SETUP}

The schematic diagram for the implemented longrange RDTS, based on a single channel receiver, is shown in Fig. 1. The pump light is provided by a pulsed fiber laser operating at $1550 \mathrm{~nm}$, with a pulse peak power of $50 \mathrm{~W}$, a pulse duration of $10 \mathrm{~ns}$ and a repetition rate of $5 \mathrm{kHz}$. By using a variable optical attenuator (VOA), the average optical power at the fiber input has been adjusted to $1.5 \mathrm{~mW}$, avoiding insurgence of fiber nonlinearities. The AS Raman

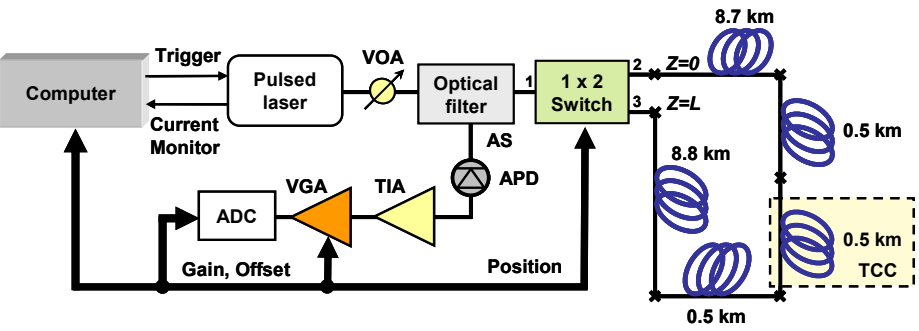

Fig. 1. Experimental setup backscattering from the sensing fiber is selected by an optical filter and detected by a high-sensitivity receiver. The receiver consists of a low-noise avalanche photodiode (APD) followed by a transimpedance amplifier (TIA), a proper variable gain amplification stage (VGA) and a customized high-resolution analog-to-digital converter (ADC). The sensing fiber actually is composed of 5 spools of graded-index multimode fiber (50/125 $\mu \mathrm{m}$ core/cladding diameter), for a total distance of $19 \mathrm{~km}$. One section of the fiber $(\sim 500 \mathrm{~m})$ at $\sim 9-\mathrm{km}$ distance has been placed in a temperaturecontrolled chamber (TCC), allowing for an accurate sensor characterization. Measurements in loop configuration are performed by using a $1 \times 2$ optical switch, which is connected to both fiber ends allowing pulses to be alternately sent in both forward and backward directions. The whole system is monitored by a computer; thus, all control signals (laser trigger, switch position, receiver gain and offset) are provided by a proper communication bus connected to the different components. Input signals (i.e. laser current monitor and AS traces) are acquired and processed in real-time.

\section{RESULTS}

Using the experimental setup shown in Fig. 1, anti-Stokes traces have been measured in both forward and backward directions. An averaging of $100 \mathrm{k}$ traces has been carried out with a measurement time of $\sim 40 \mathrm{~s}$. Fig. 2 shows the measured forward- and backward-AS traces (after normalization of laser fluctuations) for different temperatures of the TCC (varied within the range $-10^{\circ} \mathrm{C}$ to $50^{\circ} \mathrm{C}$ ), with the rest of the sensing fiber kept at room temperature $\left(26.5^{\circ} \mathrm{C}\right)$. The corresponding geometric means in forward and backward directions is then reported in Fig. 3, showing the inherent cancelation of loss (and obviously WDL) effects; in the figure, however, the impact of different Raman anti-Stokes cross sections in different fiber spools is still evident. In order to address this issue (relevant when using different fiber spools), a reference trace at a known temperature (room temperature, $25^{\circ} \mathrm{C}$ ) has been employed, as from Eq. (5), similarly to standard RDTS, leading to a calibrated temperature profile along the fiber, as shown in Fig. 4. In this figure we can note that the proposed
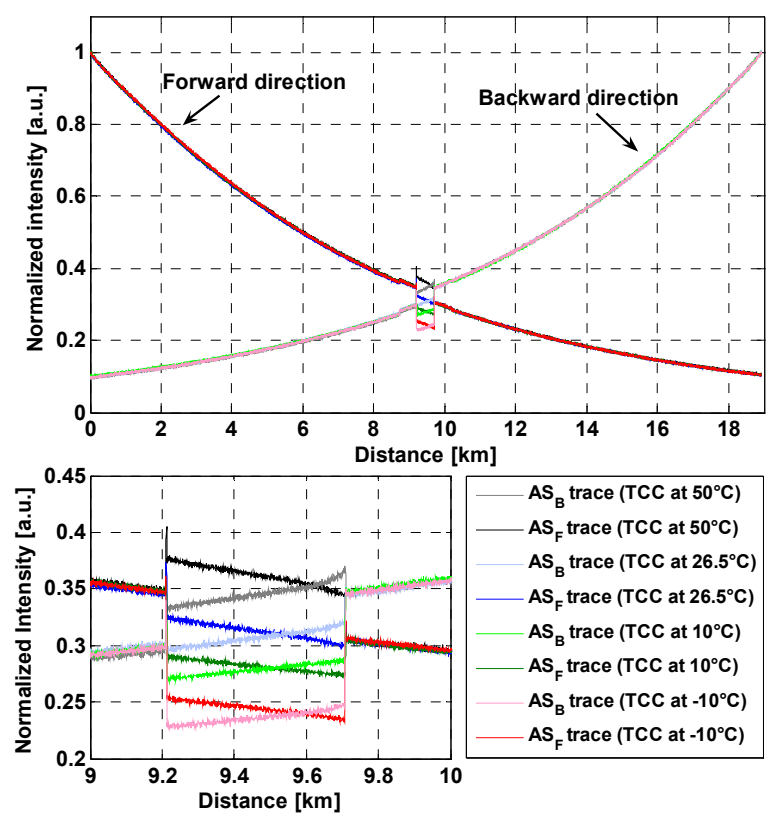

Fig. 2. Single-ended AS traces in both forward $\left(A S_{F}\right)$ and backward $\left(A S_{B}\right)$ directions for different TCC temperatures. (Top) Full normalized trace. (Bottom) Fiber spool inside TCC. 


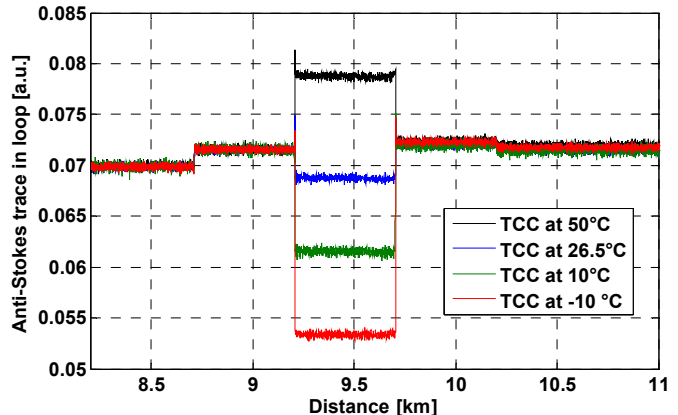

Fig. 3. Normalized AS traces for different TCC temperatures.

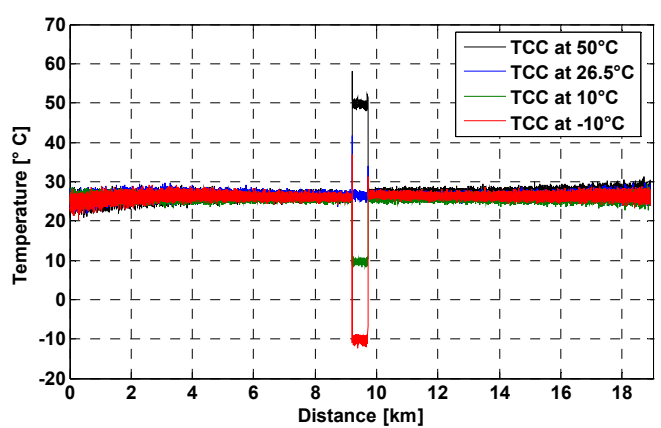

Fig. 4. Temperature profile obtained using AS traces in loop configuration for different TCC temperatures

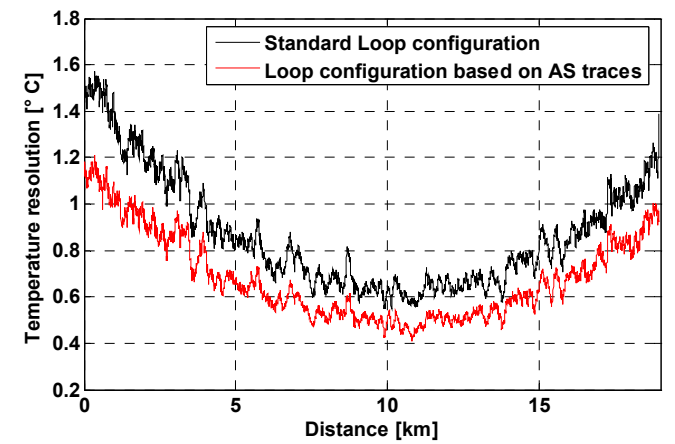

Fig. 5 Temperature resolution in loop configuration, based on anti-Stokes measurements only (red line) and standard $\mathrm{AS} / \mathrm{S}$ ratio (black line)

ports. Fig. 5 alton-loss imbalance between the two optical switch proposed technique with the one obtained with a standard RDTS in loop configuration. We can see that measurements based on AS-only loop-approach provide an enhanced temperature resolution with respect to the standard loop configuration ( 1.4 times better). This is because the temperature estimation is based on AS-intensity only, and is not affected by the additional noise introduced by Stokes intensity traces, thus allowing for an enhanced SNR $(\sim 1.3 \mathrm{~dB}$ improvement) in the final traces. The worst temperature resolution $\left(\sim 1.5^{\circ} \mathrm{C}\right)$ achieved using standard loop RDTS (occurring at the fiber input) is now improved down to $\sim 1.1^{\circ} \mathrm{C}$ with the use of AS-only loop-configuration.

In conclusion, we have demonstrated the possibility of using an AS-only approach to perform long-range distributed temperature sensing based on RDTS in loop configuration. The proposed scheme allows for the cancellation of spurious, local and wavelength-dependent loss effects employing a simple single-receiver configuration. Experimental results point out an enhanced temperature resolution ( $\sim 1.4$ times improvement $)$ compared to standard RDTS loop-schemes, thus providing a simple, cost-effective and reliable RDTS system for improved-performance long-range sensing.

\section{REFERENCES}

[1] Culshaw, B. and Kersey, A., "Fiber-Optic Sensing: A Historical Perspective," J. Lightwave Technol. 26(9), 10641078 (2008).

[2] Dakin, J. P. and Pratt, D. J., "Distributed optical fibre Raman temperature sensor using a semiconductor light source and detector," Electron. Lett. 21(13), 569-570 (1985).

[3] Bolognini, G., Park, J., Soto, M. A., Park, N. and Di Pasquale, F., "Analysis of distributed temperature sensing based on Raman scattering using OTDR coding and discrete Raman amplification," Meas. Sci. Technol. 18, 32113218 (2007).

[4] Fernandez Fernandez, A., Rodeghiero, P., Brichard, B., Berghmans, F., Hartog, A. H., Hughes, P., Williams, K. and Leach, A. P., "Radiation-Tolerant Raman Distributed Temperature Monitoring System for Large Nuclear Infrastructures," IEEE Trans. Nucl. Sci. 52(6), 2689-2691 (2005).

[5] Hwang, D., Yoon, D.-J., Kwon, I.-B., Seo, D.-C. and Chung, Y., "Novel auto-correction method in a fiber-optic distributed-temperature sensor using reflected anti-Stokes Raman scattering," Opt. Express 18(10), 9747-9754 (2010).

[6] Suh, K. and Lee, C., "Auto-correction method for differential attenuation in a fiber-optic distributed-temperature sensor," Opt. Lett. 33(16), 1845-1847 (2008). 